\title{
A task-difficulty artifact in subliminal priming
}

\author{
Michael S. Pratte and Jeffrey N. Rouder \\ University of Missouri, Columbia, Missouri
}

\begin{abstract}
Subliminal priming is said to occur when a subliminal prime influences the classification of a subsequent target. Most subliminal-priming claims are based on separate target- and prime-classification tasks. Because primes are intended to be subliminal, the prime-classification task is difficult, and the target-classification task is easy. To assess whether this task-difficulty difference accounts for previous claims of subliminal priming, we manipulated the ease of the prime-classification task by intermixing long-duration (visible) primes with short-duration (near liminal) ones. In Experiment 1, this strategy of intermixing long-duration primes raised classification of the shortduration ones. In Experiments 2 and 3, prime duration was lowered in such a way that prime classification was at chance in intermixed presentations. Under these conditions, we failed to observe any priming effects; hence, previous demonstrations of subliminal priming may simply have reflected a task-difficulty artifact.
\end{abstract}

The phenomenon of priming without awareness, in which primes escape subjective awareness but nevertheless affect subsequent behavior, has been demonstrated repeatedly in a variety of paradigms. Bargh, Chen, and Burrows (1996) serves as a suitable example: Participants waited longer to interrupt an unresponsive experimenter after unscrambling words that were related to politeness than they did after words that were related to rudeness. Likewise, participants who were exposed to words that were related to old age left the laboratory more slowly than did those who were not.

Priming without awareness, however, is not the same as subliminal priming. Subliminal primes are presented so quickly or in such impoverished conditions that they are not visible, even when full attention is deployed. Clearly, Bargh et al.'s (1996) primes were not subliminal; participants could have maintained awareness of the viewed words and adjusted their behavior as they wished. Cheesman and Merikle (1984) provided the critical distinction between priming without awareness and subliminal priming. They failed to find evidence of subliminal primes; that is, primes that were below an objectively determined threshold did not have an effect on responses to targets.

In the last decade, however, the field has seemingly reversed the Cheesman and Merikle (1984) finding, and many researchers claim to have demonstrated the most impressive form of subliminal priming - namely, that there are priming effects even for stimuli that cannot, under any circumstance, be detected or classified (see, e.g., Dehaene et al., 1998; Greenwald, Draine, \& Abrams, 1996; Kunde, Kiesel, \& Hoffmann, 2003; Van Opstal, Reynvoet, \& Verguts, 2005; see Snodgrass, Bernat, \& Shevrin, 2004, for a review). These findings of subliminal priming not only were surprising in their own right, but also influenced theories of human information processing (see, e.g., Green- wald, Abrams, Naccache, \& Dehaene, 2003), emotional processing (see, e.g., Li, Zinbarg, Boehm, \& Paller, 2008), and mental pathology (see, e.g., Dehaene et al., 2003). Although the claim of subliminal priming is influential, the demonstrations of its existence have been critiqued on statistical grounds (Dosher, 1998; Reingold \& Merikle, 1988; Rouder, Morey, Speckman, \& Pratte, 2007). In this article, we provide evidence that previous demonstrations may have been susceptible to a subtle methodological artifact that is separate from any statistical critiques.

The subliminal priming claim is conventionally supported by a paradigm with two separate tasks. In one task, participants respond to a clearly visible target. The target is preceded by a briefly presented prime (see Figure 1A), and the researcher assesses the degree to which the prime has influenced the response to the target. In the present experiments, single-digit numbers served as targets, and participants classified them as less than or greater than 5 . We refer to this task as target classification. In the second task, participants respond to the prime, and their ability to do so accurately is the main dependent measure. In the present experiments, single-digit numbers served as primes, and participants classified them as less than or greater than 5 . We refer to this task as prime classification. In this paradigm, the two tasks occur in separate blocks of trials, and the paradigm is referred to as the two-task design. In the present experiments, priming occurred when the less-than-5 status of the prime affected the less-than- 5 judgment about the target. In previous research with this task (e.g., Dehaene et al., 1998; Kunde et al., 2003), target classification was faster when the prime and target shared the same less-than-5 status (i.e., they were congruent) than when they had opposing less-than-5 status (i.e., they were incongruent). Subliminal priming occurs when there is a priming effect in target classification and

M. S. Pratte, prattems@gmail.com 
A

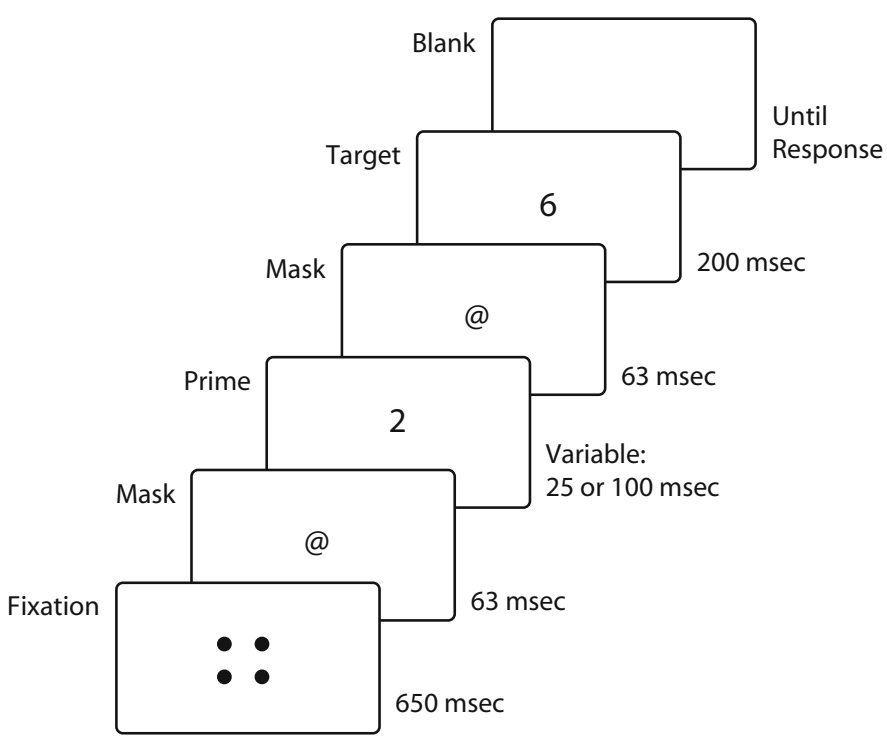

B

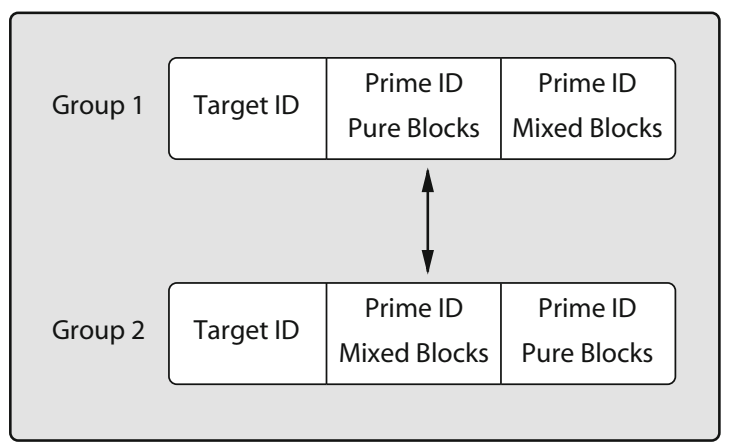

Figure 1. (A) The structure of trials in Experiment 1. The trial that is shown is incongruent (prime and target elicit opposite responses). (B) The structure of sessions. Target classification (Target ID) is followed by two prime-classification (Prime ID) tasks. Half of the participants (Group 1) performed pure-block prime classification before mixed-block prime classification; the other half (Group 2) performed the prime-classification tasks in the reverse order. The arrow indicates the between-participants comparison of interest.

prime-classification accuracy is not reliably greater than the appropriate chance baseline.

Reingold and Merikle (1988) highlighted an important assumption underlying all two-task designs: Primes must be equally visible in the prime- and target-classification tasks. The heart of our critique is that this assumption is violated. While serving as participants, we noticed that our motivation and attention waned in the primeclassification task. Simply put, it was difficult to maintain motivation and attention in such a difficult task. There is no such effect in the target-classification task. Targets are easy to classify, and it is easy to maintain attention and motivation during the task. What concerns us, then, is whether participants process primes better in the targetclassification task than in the prime-classification task as a result of differences in task difficulty. Although we experienced a motivation difference, this experience is not necessary for a task-difficulty confound. There may be other mechanisms at work, so that in difficult tasks, for example, participants adopt a speed-accuracy trade-off that is different from the one that they adopt in easy tasks, or that attention is deployed in a less ideal manner. The critical question is whether differences in overall task difficulty affect processing of the prime; fortunately, this is an empirical question.

\section{EXPERIMENT 1}

To assess the plausibility of our concern that overall task difficulty affects prime processing, we manipulated overall task difficulty by intermixing long-duration primes (100 msec) with short-duration ones $(25 \mathrm{msec})$. The long- duration primes should have been easy to classify, allowing participants to experience success, at least occasionally, thus lessening the overall difficulty of the task. Experiment 1 was designed to determine whether prime classification could be improved simply by mixing in the long-duration primes. Blocks of trials were of two types: (1) Pure blocks consisted of only short-duration primes; (2) mixed blocks consisted of both short- and long-duration primes.

The experiment used a rather conventional numberpriming design that has been used frequently to claim subliminal priming (see, e.g., Dehaene et al., 1998; Kunde et al., 2003; Naccache \& Dehaene, 2001; Van Opstal et al., 2005). In pure-block prime classification, primes were presented for $25 \mathrm{msec}$. In mixed-block prime classification, half of the primes were presented for $100 \mathrm{msec}$, and half were presented for $25 \mathrm{msec}$. Prime duration was randomized in mixed blocks, so that on any trial participants could not know whether it would be a short- or longduration prime.

\section{Method}

Participants. Forty-four University of Missouri students participated in Experiment 1 in return for credit toward a course requirement.

Stimuli and Display. The digits 2, 3, 4, 6, 7, and 8 served as targets. Primes consisted of the same digits and a blank field. Targets and primes were drawn in white against a black background in an 18-point DOS-system font. The displays were 17-in. Dell CRTs with resolutions of $800 \times 600$ pixels, refreshing at a rate of $80 \mathrm{~Hz}$. Displays were driven by Pentium 4 PCs running MS-DOS. Display and response collection was controlled by a custom-written set of C routines. ${ }^{1}$

Procedure. The structure of trials is shown in Figure 1A. Digits were randomly assigned as targets and primes, with the constraint 
that each appeared equally often as targets and as primes for each participant.

The structure of sessions is shown in Figure 1B. Sessions began with the target-classification task. Primes were presented for $25 \mathrm{msec}$. Participants were instructed to classify the target as less than or greater than 5 by depressing an assigned key on a computer keyboard. They were not informed about the existence of the primes. After performing eight target-classification blocks of 84 trials each, participants were debriefed about the existence of the primes. After debriefing, participants performed eight blocks of the prime-classification task, in which the primes were classified as greater than or less than 5. Half of the participants performed four pure blocks (prime durations of $25 \mathrm{msec}$ ) followed by four mixed blocks (prime durations of 25 and $100 \mathrm{msec}$ ). The other half of the participants performed the tasks in the reverse order. In the mixed blocks, the two durations appeared equally often and in a random order.

\section{Results}

One participant was not analyzed because of a failure to follow instructions.

Prime classification. The main question of interest was how well the short-duration 25 -msec primes were classified in pure and mixed blocks. Prior to performing the experiment, the between-participants comparison in the first prime-classification task was the primary contrast of interest (see arrowed line in Figure 1B), and the second task was added as an afterthought. ${ }^{2}$ Figure 2 shows the accuracies; the error bars are $95 \%$ confidence intervals. Indeed, for the first prime-classification task, participants classified 25 -msec primes significantly better in mixed blocks than they did in pure blocks $[t(41)=$ $3.80, p<.001]$. Also evident in the figure is a carryover

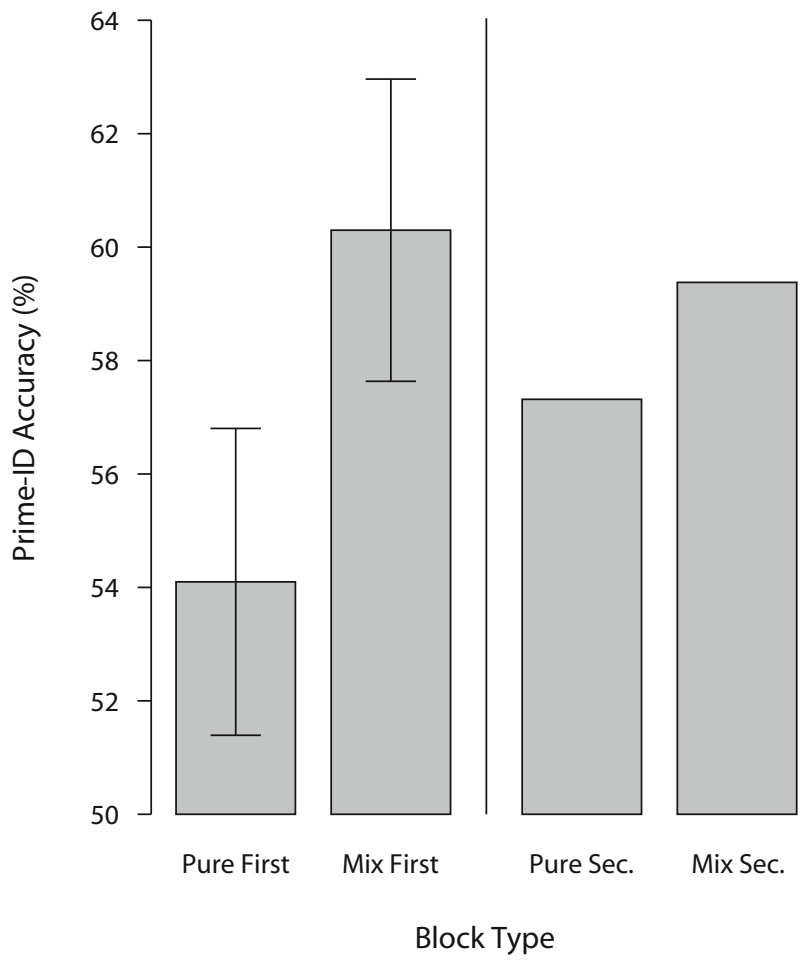

Figure 2. Prime-classification accuracies in Experiment 1. Overall, 25-msec primes were classified more accurately in mixed blocks than in pure blocks. effect: When participants observed mixed blocks first, it raised performance on subsequent pure blocks. In addition to the planned-comparisons test, we performed a post hoc mixed-effects ANOVA, with block type and order serving as factors. One element complicating this analysis was that 4 participants withdrew their consent in the second prime-classification task. We performed the preceding analysis both with these participants included and with them excluded, and this choice had no bearing on the outcome. The results of the ANOVA were a main effect of pure- versus mixed-block type $[F(1,37)=$ $17.85, p<.001]$ but, surprisingly, there was no interaction $[F(1,37)=1.39$, mean square interaction of block type by participant $=.0018]$. Finally, the 100-msec primes were classified with $80.7 \%$ accuracy, indicating that these were processed often and that they did raise overall ease.

The mixing of short- and long-duration primes clearly increased prime-classification accuracy and made the task, on the whole, easier. There may have been unintended perceptual effects of the inclusion of long-duration primes on the classification of short-duration ones, however. For instance, there may have been activation carryover from long-prime trials that enhanced the classification of subsequent short primes. In fact, perceptual carryover is known to occur in many perceptual and attention paradigms (Bertelson, 1963; Maljkovic \& Nakayama, 1994). To test for any such trial-by-trial carryover effect, we conditioned short-duration-prime classification on the previous trial's prime duration. There was no discernible difference: Performance was $59.9 \%$ for short primes that were preceded by short primes, and $60.6 \%$ for short primes that were preceded by long ones $[t(19)=0.41$, n.s.]. The difference between short-duration-prime classification in pure and mixed blocks seems to have been a global, block-long effect rather than a local, trial-by-trial effect. This course is consistent with the task-difficulty explanation of the effect of mixing primes.

Priming effect in target classification. A targetclassification trial was discarded if the response was incorrect (5\%), the response time was outside a 200 - to 1,500 -msec range $(1.1 \%)$, or the prime and target were the same digit (so as to preclude repetition priming effects). The choice of 1,500 msec as an upper truncation point on RT is conventional in this task (see, e.g., Kunde et al., 2003; Van Opstal et al., 2005). Moreover, other choices (e.g., $1 \mathrm{sec}$ or $3 \mathrm{sec}$ ) for the upper bound do not affect the results appreciably. For these data, targets that were preceded by congruent primes were classified faster than were those that were preceded by incongruent ones [521 msec for congruent primes vs. $532 \mathrm{msec}$ for incongruent primes; $t(42)=4.85, p<.0001$ ]. This 11 -msec priming effect is concordant with previous findings.

\section{Discussion}

The results of Experiment 1 show that classification of short-duration $(25-\mathrm{msec})$ primes can be increased simply by intermixing these primes with long-duration $(100-\mathrm{msec})$ ones. Because target classification is easy, it is entirely plausible that the mixed-prime-classification accuracy reflects prime processing in the target-classification 
task. In the subsequent experiments, we assessed whether subliminal priming can be observed when this measure of prime processing is used.

\section{EXPERIMENT 2}

In Experiment 2, all participants observed mixed blocks (short- and long-duration primes) during prime classification. There are two options for target classification: either short- and long-duration primes are intermixed (mixedprime target classification), or, alternatively, the primes are all short-duration (pure-prime target classification). Although we used pure-prime target classification in Experiment 1 , we did not justify this choice. Our approach in Experiment 2 was to explore the issue and choose the option that maximized the priming effect. This way, if subliminal priming did occur, we maximized our chances of detecting it; moreover, if subliminal priming did not occur, we could be confident that this null result was not the result of an injudicious choice of target-classification task.

There were two main variables in Experiment 2 that were manipulated in a between-participants fashion. One variable was target-classification-task type: Half of the participants observed pure-prime target classification, whereas the other half observed mixed-prime target classification. Second, the duration and intensity of the shortduration prime was manipulated through several levels to observe a range of near-threshold prime-classification performance. We expected that the least visible of these primes would not elicit above-chance performance.

\section{Method}

Participants. Eighty-seven University of Missouri students participated in Experiment 2 in return for credit toward a course requirement. We had planned to run an additional 73 participants, but the preliminary analysis of the experiment showed this plan to be unnecessary, as is discussed below.

Design. The experiment was a two-factor (target-classification condition by prime visibility) between-participants design, with two levels on the first factor and four levels on the second. Prime durations were $16.7,18.2$, and $20.0 \mathrm{msec}$. These upper two durations were produced by displaying the stimulus for two frames, with refresh rates of 110 and $100 \mathrm{~Hz}$, respectively. We employed two different conditions for the $16.7-\mathrm{msec}$ primes: a two-frame display at $120 \mathrm{~Hz}$, and a one-frame display at $60 \mathrm{~Hz}$. A one-frame display should be less visible than a two-frame display, since the overall intensity is less in a comparable time frame (Bloch's law; see Kahneman \& Norman, 1964). There were thus a total of four prime-visibility conditions and a total of eight separate conditions that were obtained by crossing both factors. The 87 participants were randomly assigned to one of these eight conditions.

Procedure. Experiment 2 was identical to Experiment 1, with the following exceptions: (1) A blank-field prime was not used, and (2) target- and prime-classification blocks consisted of 72 trials.

\section{Results}

Prime classification. The top panel of Figure 3 shows the prime-classification accuracies for each prime duration in the mixed-prime-classification task. At all prime durations, the primes were classified with above-chance accuracy in the mixed task, as evidenced by the $95 \%$ confidence intervals in the figure.
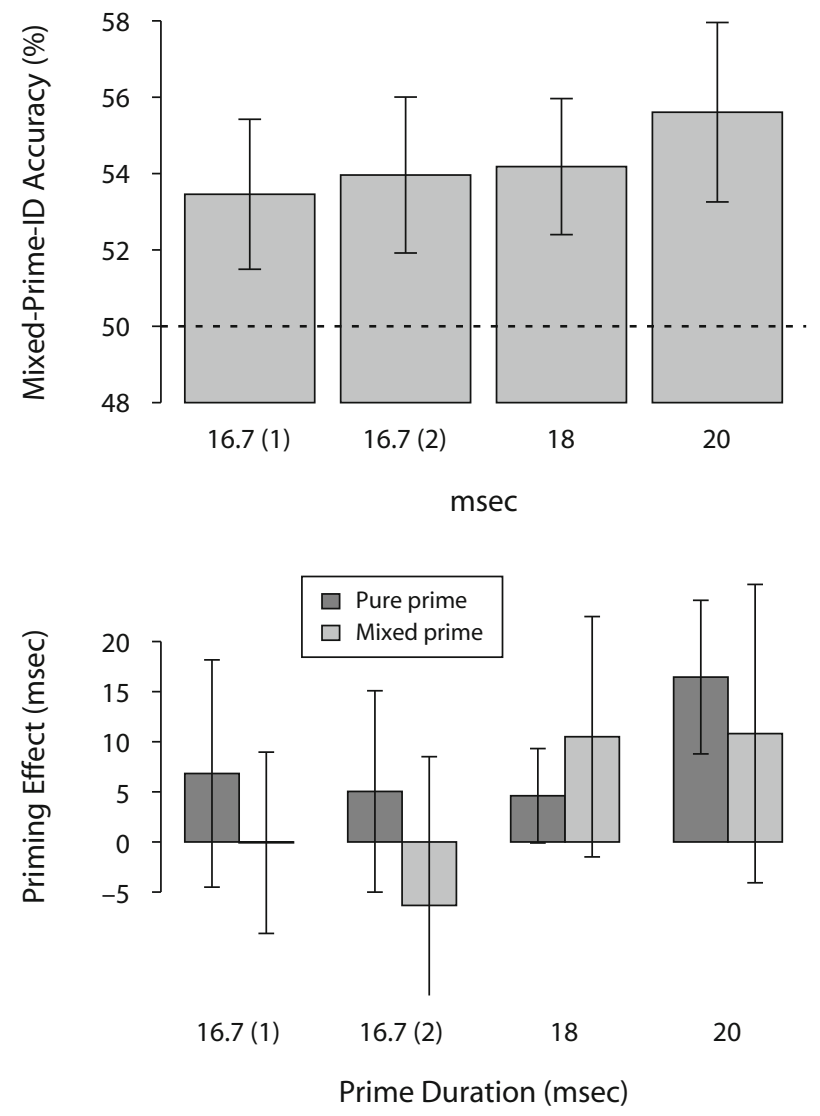

Figure 3. Results of Experiment 2. Error bars denote 95\% confidence intervals. The top panel shows mixed-prime-classification accuracies. The bottom panel shows priming effects in pure-prime and mixed-prime target-classification blocks.

Priming effect in target classification. In the targetclassification task, trials were discarded using the same criteria that were used in Experiment 1. The bottom panel of Figure 3 shows the priming effects for each prime duration in both pure-prime and mixed-prime target classification. There are two main findings. First, for the mixed-prime blocks, the priming effects for both 16.7msec presentations were below zero, even though prime classification was significantly above chance. Second, the priming effect was larger for the pure-prime condition ( 8.6 vs. $3.7 \mathrm{msec})$, although this difference was not significant $[F(1,79)=1.82, p \approx .18]$.

\section{Discussion}

Experiment 2 provided two important results. First, subliminal priming did not appear to hold for mixed-prime target presentation; that is, it was possible to achieve above-chance levels of performance with no priming effect. Second, priming in pure-prime target classification was as large or larger than priming in mixed-prime target classification. This result is concordant with Smith, Besner, and Miyoshi (1994), who found that intermixing 280-msec primes with 84-msec ones reduced the priming effect for the 84-msec primes relative to when these primes were presented alone. Although we ruled out subliminal priming 
for mixed-prime target presentations, it is still possible that there was subliminal priming for pure-prime target presentations. Our data for this case showed priming, although the primes were visible. In the next experiment, therefore, we presented the primes for even shorter durations to determine whether priming abated before primes were subliminal, or whether accuracy abated before priming did.

The fact that primes were presented so quickly and were still classified with some accuracy shows the surprisingly large magnitude of the global task-difficulty effect. In Experiment 1, 25-msec primes were classified with 54\% accuracy in pure blocks (when performed first). In Experiment 2, 16.7-msec primes were classified with the same $54 \%$ accuracy in mixed blocks. To equate performance, therefore, stimulus duration needed to be decreased by a third in mixed blocks.

\section{EXPERIMENT 3}

In Experiment 3, we assessed whether priming exists in pure-prime target classification when prime durations are so brief that primes cannot be classified with abovechance performance in mixed blocks. Experiment 3 is a near replication of Experiment 2, but with only pure-prime target classification and prime durations of $8.3 \mathrm{msec}$ (one frame at $120 \mathrm{~Hz}$ ) and $11.8 \mathrm{msec}$ (one frame at $85 \mathrm{~Hz}$ ). The two duration levels were run in a between-participants design.

\section{Method}

Forty University of Missouri students participated in Experiment 3 in return for credit toward a course requirement. Participants were randomly assigned to one of the two short-duration prime conditions. All other aspects of Experiment 3 were identical to those of Experiment 2.

\section{Results}

Prime classification. The top panel of Figure 4 shows the prime-classification accuracies for the 8.3- and 11.8 -msec primes. The 8.3 -msec primes were classified with $49.7 \%$ accuracy, which did not differ from chance performance $[t(20)=0.33, p \approx .75]$. Surprisingly, the 11.8 -msec primes were classified with $53.1 \%$ accuracy, which did differ significantly from chance performance $[t(18)=3.26, p<.01]$.

Priming effect in target classification. In the targetclassification task, trials were discarded using the same criteria that were used in Experiment 1. The bottom panel of Figure 4 shows the priming effects for the 11.8- and 8.3-msec primes; importantly, the priming effect was below zero for each. The upper $95 \%$ confidence intervals on the priming effects for 8.3 - and $11.8-\mathrm{msec}$ primes suggest that the true priming effects were no larger than 1.5 and $4.7 \mathrm{msec}$, respectively. There was sufficient power to detect a 5-msec priming effect had it existed.

Because the above results are null results, it is appropriate to worry about the sensitivity of the paradigm as well as the sensitivity of the measurements. Greenwald and colleagues (Draine \& Greenwald, 1998; Greenwald et al., 1996) have shown that near-liminal priming effects
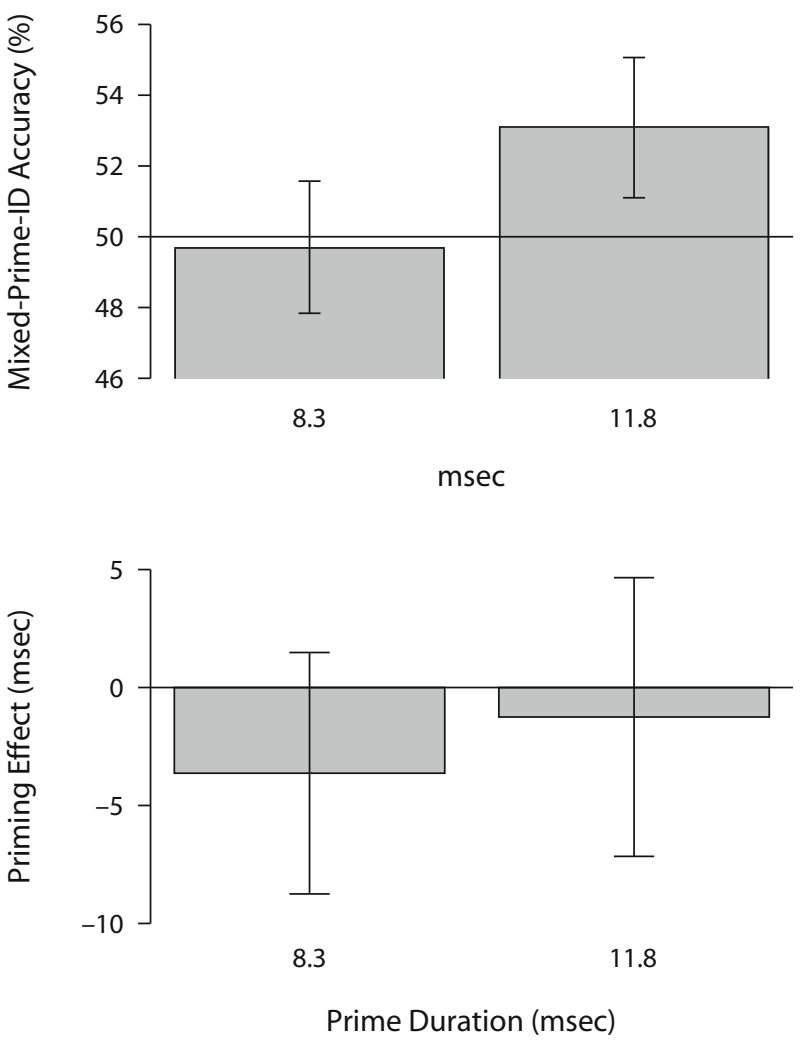

Figure 4. Results of Experiment 3. Error bars denote 95\% confidence intervals. The top panel shows mixed-prime-classification accuracies. The bottom panel shows priming effects in pure-prime target classification.

are largest for fast responses and decrease as responses slow. To maximize priming effects, they recommended using a response-window approach in which participants are forced to classify the target within approximately $300 \mathrm{msec}$. The basic motivation behind this manipulation is that the priming effect may be short-lived and thus may be attenuated by the time that participants respond in unspeeded conditions. When participants are speeded by the deadline, the prime may be more salient. We note that most previous demonstrations of subliminal priming did not rely on this method. Nonetheless, in case there was short-lived priming, we analyzed the fastest responses, which typically show the largest priming effects (Greenwald et al., 2003). To do so, we calculated priming effects at the 10th percentile of responses for each participant in each condition. The results of this analysis are concordant with those of the mean effects: Priming at the 10th percentile was not significant for the 11.8 -msec primes $[-0.7 \mathrm{msec}, t(18)=0.28, p \approx .79]$ or for the $8.3-\mathrm{msec}$ primes $[-1.4 \mathrm{msec}, t(20)=0.77, p \approx .45]$.

\section{Discussion}

The results for the 11.8-msec-prime condition provide the critical evidence that subliminal priming does not exist when task difficulty is controlled. In this case, the priming effect was nonsignificant, even though short-duration primes were classified with significantly above-chance 
accuracy. The 8.3-msec primes, which were subliminal, also produced no priming. These results suggest that when primes are at or slightly above the threshold of classifiability, there is no priming once task difficulty is controlled.

\section{GENERAL DISCUSSION}

In this article, we have argued that previous demonstrations of subliminal priming using the two-task paradigm were subject to a task-difficulty artifact. In Experiment 1, the classifiability of short-duration primes increased when the overall task was made easier through the intermixing of short- and long-duration primes. When the task-difficulty artifact was controlled for in Experiments 2 and 3, we found no evidence of subliminal priming.

\section{Previous Studies With \\ Mixed-Prime Presentations}

There are studies that have used mixed-prime presentations in prime classification and still claimed subliminal priming (Draine \& Greenwald, 1998; Naccache \& Dehaene, 2001). Draine and Greenwald used words and nonwords as primes, and they crossed these with durations of 17,33 , and 50 msec. It is not clear, however, whether these 50-msec primes were sufficiently easy to control for a task-difficulty artifact. Participants were not overly accurate with 50-msec primes; the average $d^{\prime}$ was less than .5 , and the corresponding accuracy was less than $60 \%$.

The more challenging result, at least at first glance, comes from Experiment 1 from Naccache and Dehaene (2001). These researchers intermixed 200-msec primes with 43-msec primes in their prime-detection task and still found priming. We can safely assume that the 200-msec primes were visible and controlled task difficulty. Naccache and Dehaene used a sophisticated data-analytic technique-Greenwald, Klinger, and Schuh's (1995) regression method - to claim subliminal priming. We argue below that their analysis with this method was suspect.
In the Greenwald et al. (1995) regression method, the priming effect is regressed onto prime visibility (measured by $d^{\prime}$ sensitivity). Subliminal priming is claimed when the intercept of the regression line is significantly above zero (see Figure 5A). One seminal critique of this method is that regression analysis assumes no measurement error in the regressor, or the prime visibility in this case. When the measurement of prime visibility is variable, this unaccounted variability leads to an underestimation of slope and an overestimation of intercept (Dosher, 1998). Statistical methods have been developed to address this problem (Klauer, Draine, \& Greenwald, 1998; cf. Miller, 2000), but these were not used by Naccache and Dehaene (2001). The critical question is whether Naccache and Dehaene's intercept was truly above zero, indicating a conflict with our results, or was artifactually above zero due to measurement error in the regressor.

Naccache and Dehaene (2001) assessed prime visibility by having 18 participants perform 32 prime-identification trials each. With so few trials per participant, there is excessive variability in prime-accuracy estimates and, consequently, excessive and systematic upward bias in intercept. We provide an example of this variability and bias in Figure $5 \mathrm{~B}$. The points are noisy samples from a trajectory with no subliminal priming (gray line). The variance in prime visibility is from the natural binomial noise that results from having only 32 prime-detection trials per participant, the number that was used by Naccache and Dehaene. The resulting regression line (black line) is far too shallow, and the intercept is far too high. In this case, in fact, it is significantly greater than zero, leading to a mistaken conclusion of subliminal priming. To show that the real Type I error rate on the test of the intercept was vastly inflated for the sample sizes in Naccache and Dehaene, we repeated the simulation in Figure 5B 1,000 times. The null hypothesis that the intercept is zero was mistakenly rejected for 994 of these 1,000 replicates, with a nominal Type I error rate of $\alpha<.05$. That is, even though the reported Type I error rate was .05, the real Type I error rate was .994! Figure 5C
A

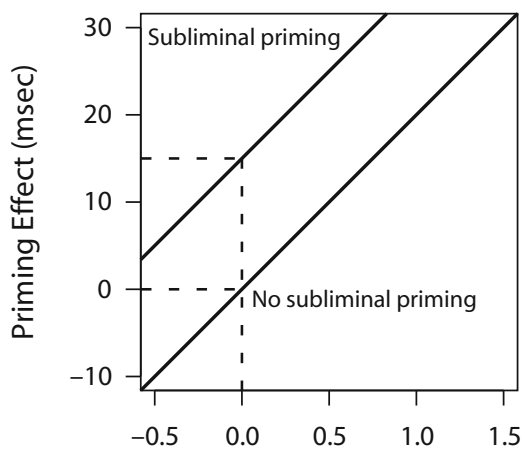

Sensitivity $\left(d^{\prime}\right)$
B

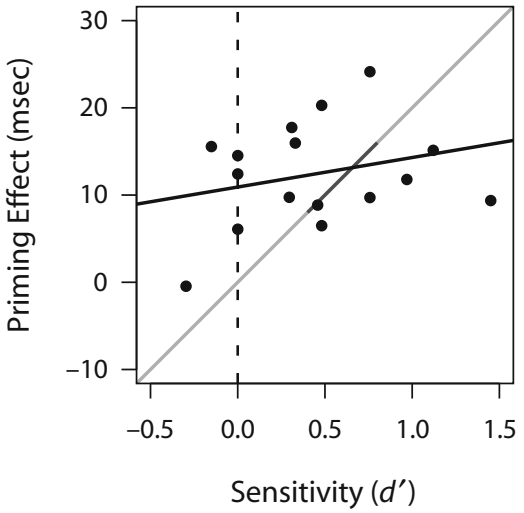

C

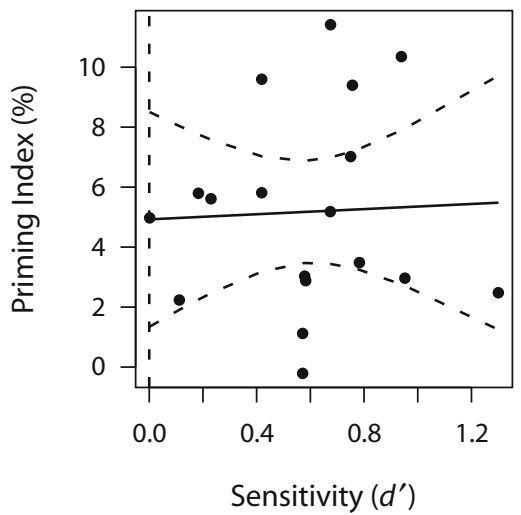

Figure 5. Variability and the Greenwald, Klinger, and Schuh (1995) regression method. (A) Relationships with and without subliminal priming. (B) Sample from a relationship without subliminal priming (dark portion of the gray line). Sample sizes and parameter values are based on Naccache and Dehaene's (2001) Experiment 1. The black line shows the regression analysis for the sampled data, which incorrectly suggests a positive intercept. (C) Results of Naccache and Dehaene's Experiment 1 (see their Figure 2, p. 220). 
shows Naccache and Dehaene's data, and the potential for the artifact is obvious. Therefore, Naccache and Dehaene provided no credible evidence for subliminal priming and their results pose no challenge to ours.

\section{The Interpretation of Mixed-Prime Presentations}

In this article, we have shown that mixing shortduration primes with long-duration ones raises classification performance for the short-duration primes. We attribute this effect to increases in global levels of motivation or attention during the task. Because participants have some chance of being correct, they are engaged in the task, much as they are in target classification. An alternative interpretation is possible, however. According to this interpretation, participants may pay greater attention to primes in prime classification than in target classification, since the former requires prime processing. In this interpretation, prime classification inherently overestimates the prime processing in target classification. The intermixing of long-duration primes exacerbates this overestimation, moreover, since the long-duration primes allow participants to more skillfully direct attention to the short-duration primes. According to this view, in the present study we have unartfully stacked the deck against subliminal priming.

This alternative interpretation is unappealing for several reasons. First, participants did show priming effects for prime durations as fast as $18 \mathrm{msec}$; therefore, there is evidence that primes were being processed during target classification. Moreover, the notion that participants can selectively attend to one brief temporal window (the target) and not attend to an immediately adjacent temporal window (the prime) at the same location is undocumented. Most theories of attention have a temporal component (e.g., Sperling \& Weichselgartner, 1995) in that attention cannot be turned on and off so rapidly. We suspect that the fixation cross drew attention, which was heightened with the quick changes in the mask-prime-mask-target ensemble. The critique might be more viable in a design in which the target was displayed until response. In such a design, a participant could conceivably look away from the stimuli for a second and still perform the task. In our design, however, targets were displayed for $200 \mathrm{msec}$, and it seems unlikely that attention could have been deployed so accurately over time to reliably isolate the target from the prime. All said, there simply is no evidence that participants can selectively attend to the prime in prime classification but not in target classification. With the selective-attention interpretation discounted, the results of the reported experiments showed no evidence for subliminal priming and suggest that previous demonstrations were artifacts of task difficulty.

Although we do not believe that the selective-attention argument carries weight, it is useful to consider its ramifications should it hold. The presence of selective attention does not mitigate the possibility that global motivation factors affect prime-classification performance; hence, researchers can never know whether prime processing is better or worse in prime categorization than in target cate- gorization. It may be better because of selective attention, or it may be worse due to global motivational differences. Perhaps, then, the minimal conclusion that can be drawn from our work is that two-task designs are ill-suited for assessing subliminal priming, and previous demonstrations with these designs are suspect.

Although we found no evidence of subliminal number priming, this null result does not necessarily generalize to other domains, such as arrow priming (see, e.g., Eimer \& Schlaghecken, 2002; Vorberg, Mattler, Heinecke, Schmidt, \& Schwarzbach, 2003) or repetition priming (see, e.g., Dehaene et al., 2001). Moreover, perhaps subliminal priming will appear at even lower prime durations than the 8 msec that was used here (see Snodgrass et al., 2004, for an alternative that anticipates such a result). Rather, our results in number priming serve as a general warning that any findings with the two-task paradigm are tenuous. We end by reiterating the advice of Reingold and colleagues (e.g., Reingold, 2003, 2004; Reingold \& Merikle, 1988, 1990) that the two-task paradigm is to be avoided, because it is difficult to know whether prime processing has been equated across the two tasks.

\section{AUTHOR NOTE}

This research is supported by NSF Grant SES- 0351523 and by NIMH Grant R01-MH071418. We thank Anthony Greenwald, Sean Vecera, and Michael Snodgrass for insightful comments. Correspondence concerning this article should be addressed to M. S. Pratte, Department of Psychological Sciences, 210 McAlester Hall, University of Missouri, Columbia, MO 65211 (e-mail: prattems@gmail.com).

\section{REFERENCES}

Bargh, J. A., Chen, M., \& Burrows, L. (1996). Automaticity of social behavior: Direct effects of trait construct and stereotype activation on action. Journal of Personality \& Social Psychology, 71, 230-244.

BERTELSON, P. (1963). S-R relationships and reaction times to new versus repeated signals in a serial task. Journal of Experimental Psychology, 65, 478-484.

Cheesman, J., \& Merikle, P. M. (1984). Priming with and without awareness. Perception \& Psychophysics, 36, 387-395.

Dehaene, S., Artiges, E., Naccache, L., Martelli, C., Viard, A., SCHÜRHOFF, F., ET AL. (2003). Conscious and subliminal conflicts in normal subjects and patients with schizophrenia: The role of the anterior cingulate. Proceedings of the National Academy of Sciences, 100, 13722-13727.

Dehaene, S., Naccache, L., Cohen, L., Le Bihan, D., Mangin, J.-F., Poline, J.-B., \& RiviéRe, D. (2001). Cerebral mechanisms of word masking and unconscious repetition priming. Nature Neuroscience, 4, $752-758$.

Dehaene, S., Naccache, L., Le Clec'H, G., Koechlin, E., MuelLer, M., Dehaene-Lambertz, G., ET AL. (1998). Imaging unconscious semantic priming. Nature, $\mathbf{3 9 5}, 597-600$.

Dosher, B. A. (1998). The response-window regression method-Some problematic assumptions: Comment on Draine and Greenwald (1998). Journal of Experimental Psychology: General, 127, 311-317.

Draine, S. C., \& Greenwald, A. G. (1998). Replicable unconscious semantic priming. Journal of Experimental Psychology: General, 127, 286-303.

Eimer, M., \& Schlaghecken, F. (2002). Links between conscious awareness and response inhibition: Evidence from masked priming. Psychonomic Bulletin \& Review, 9, 514-520.

Greenwald, A. G., Abrams, R. L., Naccache, L., \& Dehaene, S. (2003). Long-term semantic memory versus contextual memory in unconscious number processing. Journal of Experimental Psychology: Learning, Memory, \& Cognition, 29, 235-247. 
Greenwald, A. G., Draine, S. C., \& Abrams, R. L. (1996). Three cognitive markers of unconscious semantic activation. Science, 273, 1699-1702.

Greenwald, A. G., Klinger, M. R., \& Schuh, E. S. (1995). Activation by marginally perceptible ("subliminal") stimuli: Dissociation of unconscious from conscious cognition. Journal of Experimental Psychology: General, 124, 22-42.

Kahneman, D., \& Norman, J. (1964). The time-intensity relation in visual perception as a function of observer's task. Journal of Experimental Psychology, 68, 215-220.

Klauer, K. C., Draine, S. C., \& Greenwald, A. G. (1998). An unbiased errors-in-variables approach to detecting unconscious cognition. British Journal of Mathematical \& Statistical Psychology, 51, 253-267.

Kunde, W., Kiesel, A., \& Hoffmann, J. (2003). Conscious control over the content of unconscious cognition. Cognition, 88, 223-242.

Li, W., Zinbarg, R. E., Boehm, S. G., \& Paller, K. A. (2008). Neural and behavioral evidence for affective priming from unconsciously perceived emotional facial expressions and the influence of trait anxiety. Journal of Cognitive Neuroscience, 20, 95-107.

MaljKovic, V., \& NaKayama, K. (1994). Priming of pop-out: I. Role of features. Memory \& Cognition, 22, 657-672.

Miller, J. (2000). Measurement error in subliminal perception experiments: Simulation analyses of two regression methods. Journal of Experimental Psychology: Human Perception \& Performance, 26, 1461-1477.

Naccache, L., \& Dehaene, S. (2001). Unconscious semantic priming extends to novel unseen stimuli. Cognition, 80, 215-229.

ReIngold, E. M. (2003). Interpreting dissociations: The issue of task comparability. Cortex, 39, 174-176.

Reingold, E. M. (2004). Unconscious perception and the classic dissociation paradigm: A new angle? Perception \& Psychophysics, 66, 882-887.

Reingold, E. M., \& Merikle, P. M. (1988). Using direct and indirect measures to study perception without awareness. Perception \& Psychophysics, 44, 563-575.

Reingold, E. M., \& Merikle, P. M. (1990). On the inter-relatedness of theory and measurement in the study of unconscious processes. Mind \& Language, 5, 9-28.

Rouder, J. N., Morey, R. D., Speckman, P. L., \& Pratte, M. S. (2007). Detecting chance: A solution to the null sensitivity problem in subliminal priming. Psychonomic Bulletin \& Review, 14, 597-605.

Smith, M. C., Besner, D., \& Miyoshi, H. (1994). New limits to automaticity: Context modulates semantic priming. Journal of Experimental Psychology: Learning, Memory, \& Cognition, 20, 104-115.

Snodgrass, M., Bernat, E., \& Shevrin, H. (2004). Unconscious perception: A model-based approach to method and evidence. Perception \& Psychophysics, 66, 846-867.

Sperling, G., \& Weichselgartner, E. (1995). Episodic theory of the dynamics of spatial attention. Psychological Review, 102, 503-532.

Van Opstal, F., Reynvoet, B., \& Verguts, T. (2005). How to trigger elaborate processing? A comment on Kunde, Kiesel, and Hoffmann (2003). Cognition, 97, 89-97.

Vorberg, D., Mattler, U., Heinecke, A., Schmidt, T., \& SchwarzBACH, J. (2003). Different time courses for visual perception and action priming. Proceedings of the National Academy of Sciences, 100, 6275-6280.

\section{NOTES}

1. In experiments like these, it is critical that the display system obey all timing requirements without missing a refresh. The accuracy of our system was tested by placing a photodiode over the display and recording voltage output on a separate computer. Our system did not skip a refresh command during $10 \mathrm{~h}$ of continuous presentation of experimental trials (18,000 trials).

2. These experiments were performed in partial fulfillment of the first author's master's thesis. In accordance with department policy, a plan of statistical analysis was submitted before the experiments were executed. In this plan, the first author specified the contrast that is highlighted in Figure 1B (arrows) to mitigate potential carryover effects.

(Manuscript received November 3, 2008; revision accepted for publication March 23, 2009.) 